\title{
Predictive Role of Neutrophil Gelatinase-Associated Lipocalin in Early Diagnosis of Platin-Induced Renal Injury
}

\author{
Mehmet Metin Seker ${ }^{*}$, Koksal Deveci², Ayse Seker ${ }^{3}$, Enver Sancakdar ${ }^{2}$, Ali \\ Yilmaz $^{4}$, A Kerim Turesin ${ }^{4}$, Turgut Kacan ${ }^{1}$, Nalan A Babacan ${ }^{1}$
}

\begin{abstract}
Background: Acute kidney injury is an important issue in chemotherapy receiving patients an neutrophil gelatinase-associated lipocalin has been proposed as a novel marker. We here aimed to assess the role of urinary levels for assessment after platin exposure. Materials and Methods: Patients who had treated with cisplatin or carboplatin or oxaliplatin containg regimens were included in this study. Baseline and postchemotherapy serum urea, creatinine, urine neutrophil gelatinase-associated lipocalin and urine creatinine levels were determined. To avoid the effects of hydration during chemotherapy infusion the urinary neutrophil gelatinase-associated lipocalin/urine creatinine ratio was used to determine acute kidney injury. Results: Of a total of 42 patients receiving platin compounds, $14(33.3 \%)$ received cisplatin containing regimens, $14(33.3 \%)$ received carboplatin and $14(33.3 \%)$ oxaliplatin. The median age was $60(37-76)$ years. Nineteen of the patients $(45.2 \%)$ had lung cancer, $12(28.6 \%)$ colorectal cancer and $11(26.2 \%)$ others. The median pre and post chemotherapy urine neutrophil gelatinase-associated lipocalin/urine creatinin ratio was $15.6 \mathrm{ng} / \mathrm{mg}$ and $35.8 \mathrm{ng} / \mathrm{mg}(\mathrm{p}=0.041)$ in the cisplatin group, $32.5 \mathrm{ng} / \mathrm{mg}$ and $86.3 \mathrm{ng} / \mathrm{mg}(\mathrm{p}=0.004)$ in the carboplatin group and $40.9 \mathrm{ng} / \mathrm{mg}$ and $62.3 \mathrm{ng} /$ $\mathrm{mg}(\mathrm{p}=\mathbf{0 . 2 4 3})$ in the oxaliplatin group. Conclusions: Nephrotoxicity is a serious side effect of chemotherapeutic agentslike cisplatin and carbopaltin, but only to a lower extent oxaliplatin. All platin compounds must be used carefully and urine neutrophil gelatinase-associated lipocalin measurement seems to be promising in detecting acute kidney injury earlier than with creatinine.
\end{abstract}

Keywords: Neutrophil gelatinase-associated lipocalin - cancer - platin chemotherapy - nephrotoxicity

Asian Pac J Cancer Prev, 16 (2), 407-410

\section{Introduction}

Cancer is one of the most common causes of deaths worldwide (Brawley, 2011; Jemal et al., 2011; Siegel et al., 2011). With the developments in the treatment modalities and newly developed chemotherapeutic agents, both the survival rates of the patients and the side effects have increased (Berry et al., 2005; Smith et al., 2009).

Nephrotoxicity is a common and important side effect of the chemotherapeutic agents. Cisplatin, carboplatin, gemcitabine, cyclophosphamide, ifosfamide, bevacizumab, and sunitinib are commonly used agents that cause nephrotoxicity via different mechanisms (Launay-Vacher et al., 2007; Lichtman et al., 2007). Nowadays, the diagnosis of renal insufficiency is based on serum creatinine level and glomerular filtration rate. But serum creatinine is not an ideal marker for many reasons (Devarajan, 2008; Nickolas et al., 2008; Parikh and Devarajan, 2008). First, serum creatinine is influenced by many factors, such as gender, muscle mass, hydration, and tubular functions. Second, serum creatinine increases 2448 hours after an injury and cannot reflect an acute injury (Haase et al., 2010). We therefore need more sensitive and rapid markers. For example, neutrophil gelatinaseassociated lipocalin (NGAL) is a $25-\mathrm{kDa}$ protein. It is rapidly and markedly released from the distal nephrons (Haase et al., 2010). Its urinary and plasma concentrations directly reflect the severity and the duration of a renal injury.

NGAL may be helpfull in differentiating prerenal disease from renal disease (Mishra et al., 2003; Bennett et al., 2008; Haase et al., 2009). It also returns to normal limits after the attenuation of the injury (Haase et al., 2009). Both plasma and urinary NGAL levels can easily be measured. In the present study, we aimed to determine the role of NGAL in predicting nephrotoxicity in patients receiving one of three different types of platinum compounds.

${ }^{1}$ Medical Oncology Department, ${ }^{2}$ Biochemistry Department, ${ }^{4}$ Internal Medicine Department, Faculty of Medicine, Cumhuriyet University, ${ }^{3}$ Public Health Directorate, Sivas, Turkey*For correspondence: mmetinseker@yahoo.com.tr 


\section{Materials and Methods}

This study includes cancer patients older than 18 years who were treated with platinum analogs at Cumhuriyet University Medical Faculty's Medical Oncology Department. Institutional ethical committee approval was obtained for this study. Patients' ages, genders, primary tumors, tumor stages, types of chemotherapy regimens, complete blood count parametres, and liver function tests parameters were recorded. Tumor stages were determined according to the TNM Classification of Malignant Tumours ( $7^{\text {th }}$ edition) (TNM) staging system.

All patients' pretreatment blood and urine samples were collected to detect pretreatment serum urea, serum creatinine, urine NGAL and urine creatinine levels. Premedication with a serotonin (5HT-3) receptor antagonist, dexamethasone, ranitidine, and antihistaminic were given to all patients. The chemotherapy infusions were performed. During the premedication and chemotherapy infusion, the average amount of fluid infused into a patient was 2500-3000 cc.

After the chemotherapy infusion, all patients' blood and urine samples were collected again to detect posttreatment serum urea, and creatinine and urine NGAL levels.

Urine samples for NGAL and creatinine were collected from all the individuals and obtained samples were stored at $-70^{\circ} \mathrm{C}$ until the time of analysis. Urine creatinine measurement was carried out using Jaffe method and Simens Advia-2400 equipment (Siemens Medical Solutions Diagnostics, Tarrytown, USA.). Urine NGAL was measured by chemiluminescent microparticle immunoassay (CMIA) method using Architect i2000sr Immunoassay Analyzer (Abbott Ireland Diagnostics Division Lisnamuck, Longford Co. Longford, Ireland). Intraassay $\mathrm{CV}$ value was \%3.74 \pm 0.74 and interassay $\mathrm{CV}$ was $\% 2.1 \pm 3.19$ for uNGAL.

Mean values and standard deviations were calculated and statistical significance of the differences between groups was evaluated. A computer program (SPSS version 14.0, Chicago, IL, USA) was used for statistical analysis. Data were analyzed by Wilcoxon test (Nonparametric version of paired samples T-test). All hypotheses tested using a criterion level of $\mathrm{p}=0.05$.

\section{Results}

A total of 42 patients were included in this study. Of these, $22(52.4 \%)$ were male and $20(47.6 \%)$ were female. The median age was 60 years (37-76 years). Nineteen patients $(45.3 \%)$ had lung cancer, 12 (28.6\%) colorectal cancer, and 11 (26.2\%) other cancers (Table 1). Fourteen patients $(33.3 \%)$ were treated with cisplatinbased chemoregimens, 14 (33.3\%) with carboplatinbased regimens, and 14 (33.3\%) with oxaliplatin-based regimens.

The median pretreatment urine NGAL level was $26.2 \pm 39.5 \mathrm{pg} / \mathrm{ml}$ and the posttreatment urine NGAL level was $11.3 \pm 239.1 \mathrm{pg} / \mathrm{ml}$. The median pretreatment serum and urine creatinine levels were $0.8 \pm 0.2 \mathrm{mg} / \mathrm{dl}$ and $73.3 \pm 61.0 \mathrm{mg} / \mathrm{dl}$, respectively. The median posttreatment serum and urine creatinine levels were $0.79 \pm 0.2 \mathrm{mg} / \mathrm{dl}$ and $19.0 \pm 24.0 \mathrm{mg} / \mathrm{dl}$, respectively (Table 2). To avoid false low urine NGAL levels due to overhydration during chemotherapy infusion, urine NGAL/urine creatinine ratio were used to determine an acute renal injury.

For patients receiving cisplatin, the urine NGAL/

\section{Table 1. Patients' Baseline Characteristics}

\begin{tabular}{lr}
\hline Age & n $(\%)$ \\
\hline Gender & $60.4(37-76$ years) \\
Male & $22(52.4 \%)$ \\
Female & $20(47.6 \%)$ \\
Primary tumor & \\
Lung cancer & $19(45.2 \%)$ \\
Colorectal cancer & $12(28.6 \%)$ \\
Genitourinary cancer & $5(11.9 \%)$ \\
Gastric cancer & $2(4.8 \%)$ \\
Mesothelioma & $2(4.8 \%)$ \\
Pancreatic cancer & $2(4.8 \%)$ \\
Stage at diagnosis & \\
Non-metastatic & $23(54.8 \%)$ \\
Metastatic & $19(45.2 \%)$ \\
Chemotherapy regimen & \\
Taxanes* & $16(38.1 \%)$ \\
Fluorouracil/Capecitabine & $13(31.0 \%)$ \\
Gemcitabine & $7(16.7 \%)$ \\
Other & $6(14.3 \%)$ \\
\hline
\end{tabular}

*docetaxel or paclitaxel

Table 2. Patients' Pretreatment and Posttreatment Renal Function Test Parametres

\begin{tabular}{|c|c|c|c|c|}
\hline & S.creatinine (mg/dl) & U.creatinine (mg/dl) & U.NGAL (pg/ml) & U.NGAL/U.creatinine (ng/mg) \\
\hline \multicolumn{5}{|l|}{ Cisplatin } \\
\hline Pretreatment & $0.78 \pm 0.09$ & $95.7 \pm 72.1$ & $27.7 \pm 18.7$ & $15.6 \pm 61.4$ \\
\hline Posttreatment & $0.82 \pm 0.27$ & $13.9 \pm 17.2$ & $8.9 \pm 61.0$ & $35.8 \pm 88.9$ \\
\hline $\mathrm{p}$ value & 0.95 & 0.001 & 0.76 & 0.041 \\
\hline \multicolumn{5}{|l|}{ Carboplatin } \\
\hline Pretreatment & $0.87 \pm 0.24$ & $74.3 \pm 51.0$ & $20.8 \pm 56.9$ & $32.5 \pm 43.9$ \\
\hline Posttreatment & $0.76 \pm 0.21$ & $22.2 \pm 21.2$ & $13.4 \pm 18.8$ & $86.3 \pm 95.7$ \\
\hline $\mathrm{p}$ value & 0.32 & 0.003 & 0.053 & 0.004 \\
\hline \multicolumn{5}{|l|}{ Oxaliplatin } \\
\hline Pretreatment & $0.76 \pm 0.39$ & $59.9 \pm 58.6$ & $23.4 \pm 33.8$ & $40.9 \pm 44.3$ \\
\hline Posttreatment & $0.78 \pm 0.23$ & $20.9 \pm 30.9$ & $10.2 \pm 409.9$ & $62.3 \pm 493.4$ \\
\hline $\mathrm{p}$ value & 0.91 & 0.073 & 0.41 & 0.243 \\
\hline
\end{tabular}

*S: serum; U: urine 
urine creatinine ratio increased in 10 patients $(71.4 \%)$, decreased in three $(21.4 \%)$, and did not changed in one $(7.2 \%)$. For patients receiving carboplatin, the urine NGAL/urine creatinine ratio increased in 11 patients $(78.5 \%)$, decreased in one $(7.2 \%)$, and was unchanged in two $(14.3 \%)$. In patients receiving oxaliplatin, the urine NGAL/urine creatinine ratio increased in eight patients $(57.1 \%)$ and decreased in six (42.9\%). The differences between posttreatment and pretreatment urine NGAL/ urine creatinine ratios were not significant among patients receiving cisplatin, carboplatin, and oxaliplatin ( $p>0.05)$.

\section{Discussion}

Due to the increasing number of cancer patients, more patients are receiving chemotherapy, and unfortunately, more patients are experiencing side effects. The management of these side effects is now more important because a survival benefit can be gained with the contributions of new treatment modalities and chemetherapeutic agents.

Nephropathy is the one of the most important side effects that may influence morbidity and mortality. Drug-induced nephropathy is the fifth most common cause of acute kidney injury (AKI) (Haase et al., 2010). Nowadays, serum creatinine level and renal biopsy are used to diagnosis AKI. But a biopsy is invasive, requires a long time, and is a cost-intensive method (Haase and Mertens, 2010). Serum creatinine is not an ideal marker because it may be influenced by many factors and does not increase early on (Haase et al., 2010).

NGAL seems to be more sensitive and more rapid then serum creatinine in predicting AKI. Mishra et al. investigated the role of NGAL in the early diagnosis of AKI in children who underwent a cardiopulmonary bypass (Mishra et al., 2005). They found that NGAL increased 10-fold within two to six hours in patients who subsequently developed AKI. This finding was confirmed in adults by Wagener et al. (Wagener et al., 2006). NGAL was shown to be a biomarker of nephrotoxicity after contrast administration in several studies (BachorzewskaGajewska et al., 2006; Ling et al., 2008; Malyszko et al., 2009). In the study by Bachorzewska et al., NGAL was found to be a sensitive early biomarker of renal impairment after a percutaneous coranary intervention (Bachorzewska-Gajewska et al., 2006). Malyszko et al. investigated the role of NGAL in contrast-induced nephropathy in diabetic patients with normal serum creatinine levels who underwent a cardiac catheterization (Malyszko et al., 2009).

The role of NGAL in cisplatin-induced nephropathy has also investigated in animal and human models (Mishra et al., 2004; Gaspari et al., 2010; Lin et al., 2013). In a prospective study of 12 cancer patients who received cisplatin, NGAL increased two days after the cisplatin administration (Gaspari et al., 2010). In this study, the increase at serum creatinine level was detected 4.5 days after the administration of cisplatin. But in the study by Lin et al., the increase in NGAL level was deteced from the twelth hour of cisplatin administration (Lin et al., 2013).

In our study, we evaluated the role of NGAL in patients receiving platinum compounds at an earlier time: the second hour of chemotherapy. When assesed with serum creatinine, one-third of the patients receiving cisplatin suffered from nephrotoxicity (Lin et al., 2013). Although Kos et al., did not show a correlation between NGAL and nephrotoxicity our study showed that $71 \%$ of the patients were at risk of cisplatin-induced nephrotoxicity (Kos et al., 2013). One reason for the difference between these percentages may be that the nephrotoxicity in some patients may be transient. So we can not detect it with serum creatinine levels as it is generally measured one or more days after the cisplatin administration. Another reason may be that cisplatin-induced nephropathy is not big enough to increase serum creatinine. But these patients are at risk of developing renal insufficiency in subsequent administrations.

To date, there is no data about the role of NGAL in carboplatin-induced nephropathy. Our trial showed that carboplatin may have a nephrotoxic effect and NGAL can be used to predict nephrotoxicity in patients receiving carboplatin. Also, there is no data about the role of NGAL in patients receiving oxaliplatin. Our results may show that oxaliplatin has no nephrotoxicity, and we did not find any differences between pretreatment urine NGAL/ urine creatinine ratio and posttreatment urine NGAL/urine creatinine ratio. Perhaps oxaliplatin caused nephrotoxicity with a different mechanism and NGAL is not a suitable marker for predicting the oxaliplatins' nephrotoxicity.

In conclusion, platin-related AKI is a common side effect. Both cisplatin and carboplatin may have nephrotoxicity. Oxaliplatin seems to be more reliable in terms of nephrotoxicity, or we will still need more sensitive markers to predict oxaliplatin-induced AKI.

\section{References}

Bachorzewska-Gajewska H, Malyszko J, Sitniewska E, Malyszko JS, Dobrzycki S (2006). Neutrophil-gelatinaseassociated lipocalin and renal function after percutaneous coronary interventions. Am J Nephrol, 26, 287-92.

Bennett M, Dent CL, Ma Q, et al (2008). Urine NGAL predicts severity of acute kidney injury after cardiac surgery: a prospective study. Clin J Am Soc Nephrol, 3, 665-73.

Berry DA, Cronin KA, Plevritis SK, et al (2005). Effect of screening and adjuvant therapy on mortality from breast cancer. N Engl J Med, 353, 1784-92.

Brawley OW (2011). Avoidable cancer deaths globally. $C A$ Cancer J Clin, 61, 67-8.

Devarajan P (2008). Neutrophil gelatinase-associated lipocalin-an emerging troponin for kidney injury. Nephrol Dial Transplant, 23, 3737-43.

Gaspari F, Cravedi P, Mandala M, et al (2010). Predicting cisplatin-induced acute kidney injury by urinary neutrophil gelatinase-associated lipocalin excretion: a pilot prospective case-control study. Nephron Clin Pract, 115, 154-60.

Haase M, Bellomo R, Devarajan P, et al (2009). Accuracy of neutrophil gelatinase-associated lipocalin (NGAL) in diagnosis and prognosis in acute kidney injury: a systematic review and meta-analysis. Am J Kidney Dis, 54, 1012-24.

Haase M, Haase-Fielitz A, Bellomo R, et al (2009). Sodium bicarbonate to prevent increases in serum creatinine after cardiac surgery: a pilot double-blind, randomized controlled trial. Crit Care Med, 37, 39-47. 
Mehmet Metin Seker et al

Haase M, Haase-Fielitz A, Bellomo R, Mertens PR (2011). Neutrophil gelatinase-associated lipocalin as a marker of acute renal disease. Curr Opin Hematol, 18, 11-8.

Haase M, Mertens PR (2010). Urinary biomarkers--silver bullets to faster drug development and nephron protection. Nephrol Dial Transplant, 25, 3167-9.

Jemal A, Bray F, Center MM, et al (2011). Global cancer statistics. CA Cancer J Clin, 61, 69-90.

Kos FT, Sendur MA, Aksoy S, et al (2013). Evaluation of renal function using the level of neutrophil gelatinase-associated lipocalin is not predictive of nephrotoxicity associated with cisplatin-based chemotherapy. Asian Pac J Cancer Prev, 14, 1111-4.

Launay-Vacher V, Oudard S, Janus N, et al (2007). Prevalence of Renal Insufficiency in cancer patients and implications for anticancer drug management: the renal insufficiency and anticancer medications (IRMA) study. Cancer, 110, 1376-84.

Lichtman SM, Wildiers H, Launay-Vacher V, et al (2007). International society of geriatric oncology (SIOG) recommendations for the adjustment of dosing in elderly cancer patients with renal insufficiency. Eur J Cancer, 43, 14-34.

Lin HY, Lee SC, Lin SF, et al (2013). Urinary neutrophil gelatinase-associated lipocalin levels predict cisplatininduced acute kidney injury better than albuminuria or urinary cystatin C levels. Kaohsiung J Med Sci, 29, 304-11.

Ling W, Zhaohui N, Ben H, et al (2008). Urinary IL-18 and NGAL as early predictive biomarkers in contrast-induced nephropathy after coronary angiography. Nephron Clin Pract, 108, 176-81.

Malyszko J, Bachorzewska-Gajewska H, Poniatowski B, Malyszko JS, Dobrzycki S (2009). Urinary and serum biomarkers after cardiac catheterization in diabetic patients with stable angina and without severe chronic kidney disease. Ren Fail, 31, 910-9.

Mishra J, Dent C, Tarabishi R, et al (2005). Neutrophil gelatinase-associated lipocalin (NGAL) as a biomarker for acute renal injury after cardiac surgery. Lancet, 365, 1231-8.

Mishra J, Ma Q, Prada A, et al (2003). Identification of neutrophil gelatinase-associated lipocalin as a novel early urinary biomarker for ischemic renal injury. J Am Soc Nephrol, 14, 2534-43.

Mishra J, Mori K, Ma Q, et al (2004). Neutrophil gelatinaseassociated lipocalin: a novel early urinary biomarker for cisplatin nephrotoxicity. Am J Nephrol, 24, 307-15.

Nickolas TL, Barasch J, Devarajan P (2008). Biomarkers in acute and chronic kidney disease. Curr Opin Nephrol Hypertens, 17, 127-32.

Parikh CR, Devarajan P (2008). New biomarkers of acute kidney injury. Crit Care Med, 36, 159-65.

Siegel R, Ward E, Brawley O, Jemal A (2011). Cancer statistics, 2011: the impact of eliminating socioeconomic and racial disparities on premature cancer deaths. CA Cancer J Clin, 61, 212-36.

Smith BD, Smith GL, Hurria A, Hortobagyi GN, Buchholz TA (2009). Future of cancer incidence in the United States: burdens upon an aging, changing nation. J Clin Oncol, 27, 2758-65.

Wagener G, Jan M, Kim M, et al (2006). Association between increases in urinary neutrophil gelatinase-associated lipocalin and acute renal dysfunction after adult cardiac surgery. Anesthesiology, 105, 485-91. 